\title{
Atomistic calculations of structural and elastic properties of serpentine minerals: the case of lizardite
}

\author{
A.-L. Auzende · R. J.-M. Pellenq · B. Devouard \\ A. Baronnet $\cdot$ O. Grauby
}

\begin{abstract}
The physical properties of the hydrous phyllosilicate lizardite have been investigated by atomistic simulation using the GULP code based on transferable semi-empirical interatomic potentials. Lizardite behavior was first investigated during structure relaxation at room temperature. The Helmholtz free energy is minimum for an equilibrium structure that is in agreement with experiment. The bulk, shear, and Young modulii for lizardite were calculated along with the Poisson ratio. From the shear and bulk modulii, we also calculated translational and longitudinal acoustic wave velocities that are important quantities for tectonophysics models. As expected, lizardite is stiffer in the $a$ direction parallel to the layers than in the $c$ perpendicular direction; the variation of the unit cell parameters with pressure is in good agreement with experiment. The cohesive energy between two successive layers along $c$ direction was calculated at $0.33 \mathrm{eV}$ (i.e., $0.11 \mathrm{eV}$ per $\mathrm{OH}$ bond) in good agreement with recent ab initio calculations. Upon pressure and temperature variations, we evidenced that structural changes are mainly pressure induced; pressure being accommodated by a decrease of the $c$ parameter up to $10 \mathrm{GPa}$. We also found that the change of slope in the derivative of the $c$ cell parameter with respect to pressure occurring around $2 \mathrm{GPa}$ originates from the bending of the interlayer hydroxyl groups with respect to the layer normal direction.
\end{abstract}

A.-L. Auzende · R. J.-M. Pellenq · A. Baronnet · O. Grauby Centre de Recherche en Matière Condensée et Nanosciences, CNRS, campus de Luminy, case 913, 13288, Marseille Cedex 9, France

A.-L. Auzende $(\bowtie)$

Institut de Minéralogie et Physique des Milieux Condensés, campus Boucicaut, 140 rue de Lourmel, 75015, Paris, France

E-mail: auzende@impmc.jussieu.fr

Tel.: + 33-01-44275059

Fax: +33-01-44273485

B. Devouard

Laboratoire Magmas et Volcans,

Blaise Pascal University-OPGC-CNRS, 5, rue Kessler, 63038, Clermont-Ferrand Cedex, France
Keywords Atomistic simulation - GULP · Serpentine minerals $\cdot$ Bulk modulus

\section{Introduction}

Serpentine minerals are hydrous magnesium 1:1 phyllosilicates which can adopt various structural configurations: lizardite exhibits flat independent not chemically bonded layers (see Fig. 1), chrysotile is tubular, and antigorite shows a corrugated structure (Wicks and O'Hanley 1988). Serpentine minerals are of prime geological interest, as they are implied in several major geodynamical processes including earthquakes (Hattori et al. 2003). However, the scarcity of thermodynamic, structural, or elastic data characterizing these minerals makes difficult to predict their behavior in geological conditions. Single crystal x-ray diffraction studies (XRD) provided structural refinements for lizardite (Mellini 1982; Mellini and Zanazzi 1989; Mellini and Viti 1994; Gregorkiewitz et al. 1996) and more recently for antigorite (Capitani and Mellini 2004). However, to our knowledge, no $P-V-T$ equation of state is yet available for any of the serpentine varieties.

Computer simulations significantly contribute to a better understanding of minerals behavior. These methods are now intensively used in Earth sciences allowing predicting structure to properties relationships for minerals. Among the various approaches, atomistic calculations based on transferable semi-empirical interatomic potentials have successfully reproduced the structure and properties of many silicates (Post and Burnham 1986; Price and Parker 1988; Dove et al. 1993; Winkler et al. 1991; Archer et al. 2003) and, among them, of some phyllosilicates (Collins and Catlow 1992; Teppen et al. 1997; Sainz-Diaz et al. 2001; Palin et al. 2003). This method consists in minimizing the system (potential and/ or free) energy assuming that interactions between the atoms are fairly well described by semi-empirical interaction potentials. The significant advantage of such an approach compared to first-principle ab initio quantum 
mechanical methods is that one can compute structural, thermodynamic, and elastic properties of large systems with low symmetry (Sauer et al. 1994). Semi-empirical interatomic potentials are analytical functions that allow calculating the energy between pairs and triplets of atoms. They depend on the choice of some parameters that can be advantageously calculated using ab initio calculations in some simple cases; the transferability of such potential parameters in the case of more complex systems can then be checked or assumed.

In this paper, we report an atomistic simulation study of lizardite-1 $T$ using the General Utility Lattice Program (GULP) atomistic simulation code based on such semiempirical interatomic potentials. We optimized lizardite structure by lattice relaxation at finite pressure and temperature in order to constrain the structural evolution under geological conditions. Preexisting experimental (Mellini and Viti 1994; Mellini and Zanazzi 1989) and ab initio data (Balan et al. 2002) are used to evaluate the efficiency of the method in the case of serpentine minerals. In particular, we aim at testing the core-shell electrostatic model for ionic and iono-covalent crystals (see below) and validating the transferability of related potential parameters to the $\mathrm{Mg}, \mathrm{Si}, \mathrm{O}, \mathrm{H}$ (serpentine) system.

\section{Computational method}

Interatomic potentials

Potential energy minimization for finding an equilibrium structure consists in tracking stationary points that correspond to a minimum energy gradient with positive energy curvature (i.e., finding a set of atomic positions that minimizes system energy and give an Hessian operator with positive eigenvalues only). Such minimization procedure necessarily gives a zero temperature solution. If one aims at calculating system equilibrium properties at finite temperature then free energy calculations are to be considered. Along with Monte-Carlo or Molecular Dynamics simulations, a third possible route to study crystalline structures at a microscopic scale is the lattice dynamic theory. From the set of the system Hessian eigenvalues, one can compute the vibration partition function in the harmonic approximation and deduce all thermodynamic functions including entropy hence system free energy. Finite temperature equilibrium configuration can then be deduced by minimizing system's free energy. Free energy calculations were performed with the GULP code using the NewtonRaphson minimization method (Gale 1996, 1997; Gale and Rohl 2003). Along with the finite temperature calculations of thermodynamics properties, it allows the calculations of the elastic tensor $\left(C_{i j}\right)$. Using the elastic tensor obtained from the GULP code, further calculations of the compliance matrix $\left(S_{i j}\right)$ with our own FORTRAN90 code allow obtaining the Poisson ratio, the bulk, shear, and Young modulii (using both Reuss and Voigt definitions) and the longitudinal and transverse acoustic wave velocities.

For ionic and iono-covalent crystal structure, the GULP code uses the Born model description which considers a collection of point charges that interacts by means of electrostatic and short range semi-empirical potential functions. The electrostatic (coulombic) interactions are evaluated using the Ewald sum technique expressed by two convergent series in the real and reciprocal space and using the formal ionic charges:

$U_{\text {coul }}^{\text {recip }}=\frac{2 \pi}{V} \sum_{G} \frac{\exp \left(-G^{2} / 4 \eta\right)}{G^{2}} \sum_{i} \sum_{j} q_{i} q_{j} \exp \left(-i \vec{G} \cdot \overrightarrow{r_{i j}}\right)$

$U_{\mathrm{coul}}^{\mathrm{real}}=\frac{1}{2} \sum_{i} \sum_{j} \frac{q_{i} q_{j} \operatorname{erfc}\left(\eta^{1 / 2} r_{i j}\right)}{r_{i j}}$

where erfc is the complementary error function, $r_{i j}$ the distance between two atoms $i$ and $j, G$ a reciprocal space vector $(G \neq 0), q_{i}$ the ionic charge of atom $i . V$ is the volume of the unit cell and $\eta$ a parameter that controls the division of work between real and reciprocal space. Note that (1) the total electrostatic energy now refers to the ionic self-energy given by $U_{\text {self }}=-\sum_{i=l}^{N} q_{i}^{2}(\eta / \pi)^{1 / 2}$ that is constant in canonical conditions (fixed number $N$ of ions); (2) the choice of $\eta$ controls the number of $G$ vectors to be chosen for a given convergence accuracy, here set to $10^{-5} \mathrm{eV}$ (Gale and Rohl 2003). In modeling oxygen in silicate materials, electronic polarizability

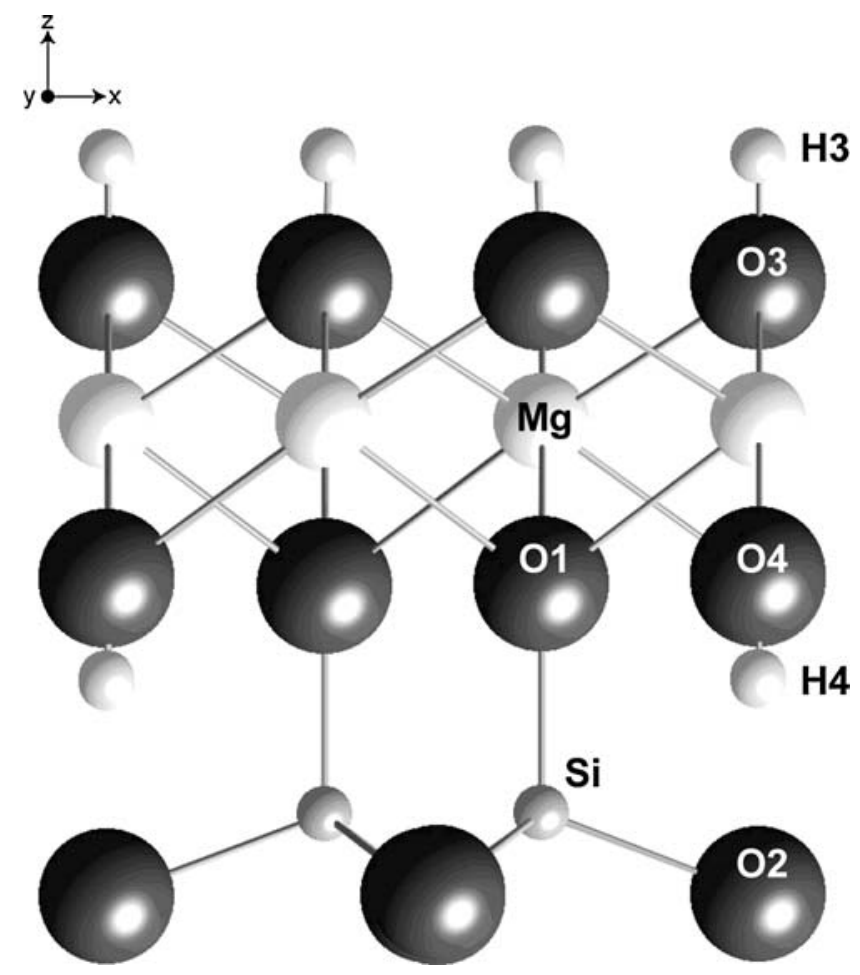

Fig. 1 Crystal structure of lizardite along (010) (Mellini and Viti 1994) 
effects are taken into account by splitting the anion into two entities: a core and a massless shell, the (formal) ionic charge $\left(q_{\mathrm{t}}=q_{\mathrm{s}}+q_{\mathrm{c}}\right)$ being shared between these two species (Dick et al. 1958). The core and the shell interact via an harmonic oscillator with a spring constant $K_{\mathrm{cs}}$. The shell polarizability is then expressed by

$Y=\frac{q_{\mathrm{s}}^{2}}{\left(K_{\mathrm{cs}}+F_{\mathrm{s}}\right)}$

where $F_{\mathrm{s}}$ is a force acting on the shell and due to the local environment. During energy minimization process, the shell is allowed to relax relatively to its core, creating a local instantaneous dipole mimicking ion polarizability.

The short-range interactions are described by a Buckingham potential which gathers an exponential repulsive and an attractive dispersive term:

$U_{\text {short }}=A_{i j} \exp \left(-\frac{r_{i j}}{\rho_{i j}}\right)-\frac{C_{i j}}{r_{i j}^{6}}$

The energy contribution of the latter term is summed within a predetermined cutoff $R_{\max }$ set to $12 \AA$ throughout this work.

When using formal ionic charges in the case of ionocovalent systems such as silicates, a three-body harmonic term has to be further considered in order to mimic the correct angle $\mathrm{O}-\mathrm{Si}-\mathrm{O}$ distribution:

$U_{3 \mathrm{~B}}=\frac{1}{2} \beta\left(\theta-\theta_{0}\right)^{2}$

where $\beta$ is an adjustable parameter and $\theta_{0}$ an equilibrium angle.

As lizardite is a hydrous phyllosilicate, the $\mathrm{O}-\mathrm{H}$ covalent interaction is simulated by a so-called Morse analytical form:

$U_{\text {Morse }}=D\left[1-\exp \left(-\alpha\left(R-R_{0}\right)\right)\right]^{2}$

where $R_{0}$ is the equilibrium position, $D$ the potential well depth, and $\alpha$ its width. All potential parameters used in this work were taken from literature and are reported in Table 1. They were optimized to describe $\mathrm{SiO}_{2}$ and $\mathrm{MgO}$ simple structures (bulk and surfaces). No further fitting to some lizardite properties was attempted in this work. Thus, the results presented here are predictions for the used potential models and related parameters.

As already mentioned, lizardite is made of a stacking of layers; each layer being constituted of one magnesium oxide octahedral sheet and one silicate tetrahedral sheet (see Fig. 1). Two crystallographic distinct $\mathrm{OH}$ groups occur in serpentine minerals. Inner $\mathrm{O}_{4}-\mathrm{H}_{4}$ bonds point towards the center of the hexagonal rings inside the layer thickness while surface $\mathrm{O}_{3}-\mathrm{H}_{3}$ lies within the interlayer space between two layers. The latter are considered to be responsible for the cohesion between two successive layers by forming hydrogen bonds. Note that from the fundamentals of interatomic or intermolecular interactions, hydrogen bonding does not result from a particular term in the interaction Hamiltonian operator that describes the system energetics. Hydrogen bonding is in fact, the consequence of the combination of terms such as electrostatics, induction, dispersion, and repulsive interactions that naturally occur in the perturbative and variational methods of quantum mechanics theory. The starting atomic positions and unit cell parameters used to model lizardite-1 $T$ are those reported from the single crystal structure refinement by Mellini and Viti (1994): the lizardite unit cell is built from a primitive cell using the $P 31 \mathrm{~m}$ space group symmetry elements; it includes three $\mathrm{Mg}$, two $\mathrm{Si}$, five $\mathrm{O}$, four $\mathrm{OH}$ (oxygen atoms that are linked to hydrogen atoms), and four $\mathrm{H}$. Note that since all oxygens are considered within the (core + shell) model, in our simulations, one unit cell of lizardite consists of 27 interactions centers. Note that all calculations in this work were done with no constrain based on space group symmetry elements nor on atomic positions in order to really check the performance of potential model (see Table 2).

\section{Thermodynamics}

System free energy can be obtained form the vibrational partition function. Helmholtz free energy writes:

$F=U_{\text {lattice }}+U_{\text {vib }}-T S_{\text {vib }}$

where $U_{\text {lattice }}$ is the minimized $0 \mathrm{~K}$ potential energy, $U_{\text {vib }}$ represents the vibrational internal energy derived in the harmonic approximation from the partition function $Z_{\text {vib }}$ calculated at the center of the Brillouin zone:

$Z_{\mathrm{vib}}=\sum_{v_{i}}\left[1-\exp \left(-\frac{h v_{i}}{k T}\right)\right]^{-1}$

where $T$ is the temperature, $k$ the Boltzmann constant, and $v_{i}$ is the lattice vibration frequencies (the histogram of such frequencies forms the so-called vibration density of state). $U_{\text {vib }}$ is then obtained:

$U_{\mathrm{vib}}=k T^{2}\left[\frac{\partial \operatorname{Ln} Z_{\mathrm{vib}}}{\partial T}\right]$

Then follows the vibrational entropy:

$S_{\mathrm{vib}}=k \ln Z_{\mathrm{vib}}+\frac{U_{\mathrm{vib}}}{T}$

The pressure $P$ in the sum of an internal contribution (phonons) with an external isostatic contribution. The internal pressure is simply obtained as

$P_{\mathrm{vib}}=k T\left[\frac{\partial \ln Z_{\mathrm{vib}}}{\partial V}\right]$

System free energy can be minimized with respect to the simulation cell volume. At convergence (equilibrium at finite temperature), the total pressure is zero. 
Table 1 Parameter values used in the interatomic potentials

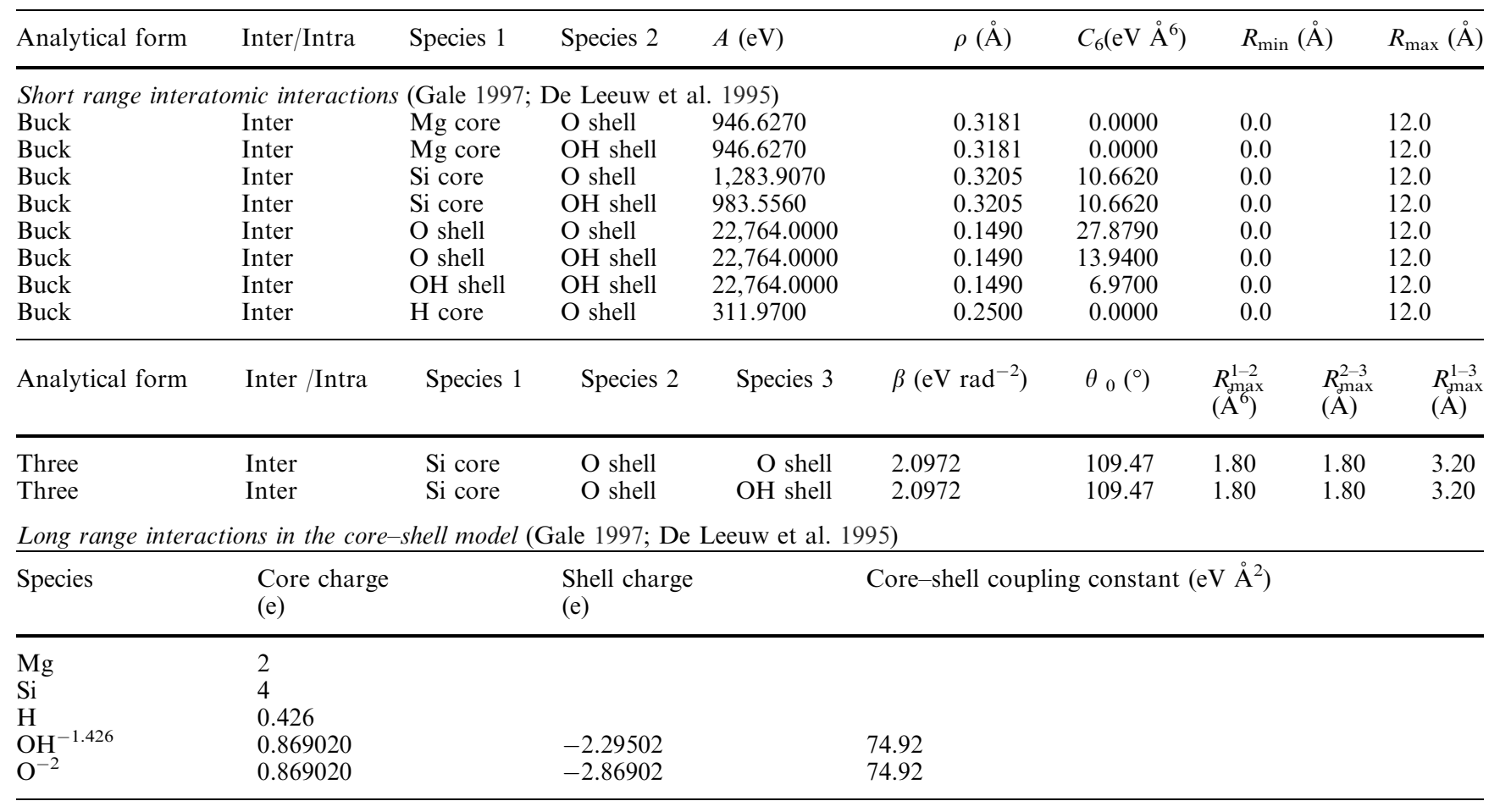

\section{Energy minimization}

The first step is finding the minimum energy structure of the present material, i.e., the local minimum on the global potential energy surface that the starting experimental coordinates lie closest to. This can be considered as a first test of the set of interatomic potential functions used to model layered magnesium silica materials such as lizardite. The task consisting in locating the global energy minimum is a far more challenging task that can be done using simulated annealing approaches combined with Monte-Carlo or Molecular Dynamics methods. Here, our strategy is a quest for the closest (local) minimum to the experimental structure using the BFGS minimization algorithm (Press et al. 1986). The practical approach taken in a BFGS optimization procedure is to initialize the Hessian matrix by performing an exact inversion of the second derivatives and then subsequently updating for a number of cycles. Criteria for convergence between successive cycles are the energy gradient norm, the change in the function (energy, enthalpy or free energy). GULP allows switching from the BFGS algorithm to the so-called rational functional optimization (RFO) when energy gradient is smaller than a disposable threshold. In the RFO method, the inverse Hessian matrix is diagonalized to obtain the eigenvalues and eigenvectors. From the eigenvalues it is possible to examine whether the matrix has the required characteristics that guarantee that a true minimum is obtained. If the number of negative eigenvalues is incorrect, then their spectrum is level-shifted to correct this and the search direction constructed appropriately until the Hessian has the right structure (only positive eigenvalues). A vibration density of state at the center of the Brillouin zone ( $\Gamma$-point) can be used as a final validation from which one gets the list of lattice vibration frequencies that should be all positive except the first three that should be zero (unit cell translational invariance).

\section{Results and discussion}

\section{Elastic properties of lizardite}

As most phyllosilicates, lizardite is expected to be softer along $c$ direction (perpendicular to the layers, see Fig. 1) compared to the other directions. Relaxations were first performed at room temperature by artificially changing each cell parameter step by step with increments of $0.05 \AA$. The free energy is calculated for each cell volume after obtaining potential energy minimum by allowing all atomic positions to relax. The minimum free energy is obtained for a unit cell volume $V_{0}$ of $184 \AA^{3}$ (see Fig. 2). The relaxed simulated structure is therefore in good agreement with available experimental data (see Table 3): for instance, the difference between the simulated and experimental cell volumes is less than $3 \%$. At first sight, the set of interatomic potentials (see Table 1) gives a fairly good description of lizardite structure. Elastic proprieties of lizardite can be estimated from the evo- 
Table 2 Relaxed fractional coordinates in lizardite unit cell

\begin{tabular}{llllll}
\hline No. & Atomic Label & Core/shell & $x$ & $y$ & $z$ \\
\hline 1 & $\mathrm{H}$ & $\mathrm{c}$ & 0.658317 & 0.999945 & 0.725929 \\
2 & $\mathrm{H}$ & $\mathrm{c}$ & 0.990734 & 0.667527 & 0.725942 \\
3 & $\mathrm{H}$ & $\mathrm{c}$ & 0.323152 & 0.332362 & 0.725942 \\
4 & $\mathrm{H}$ & $\mathrm{c}$ & 0.990734 & 0.999945 & 0.135283 \\
5 & $\mathrm{Mg}$ & $\mathrm{c}$ & 0.326618 & 0.999948 & 0.442053 \\
6 & $\mathrm{Mg}$ & $\mathrm{c}$ & 0.990738 & 0.335829 & 0.442052 \\
7 & $\mathrm{Mg}$ & $\mathrm{c}$ & 0.654847 & 0.664057 & 0.442052 \\
8 & $\mathrm{O}$ & $\mathrm{c}$ & 0.324068 & 0.666612 & 0.287454 \\
9 & $\mathrm{O}$ & $\mathrm{c}$ & 0.657401 & 0.333278 & 0.287454 \\
10 & $\mathrm{O}$ & $\mathrm{c}$ & 0.489887 & 0.999945 & 0.987327 \\
11 & $\mathrm{O}$ & $\mathrm{c}$ & 0.990735 & 0.499097 & 0.987326 \\
12 & $\mathrm{O}$ & $\mathrm{c}$ & 0.491582 & 0.500792 & 0.987326 \\
13 & $\mathrm{OH}$ & $\mathrm{c}$ & 0.658679 & 0.999945 & 0.601383 \\
14 & $\mathrm{OH}$ & $\mathrm{c}$ & 0.990735 & 0.667889 & 0.601389 \\
15 & $\mathrm{OH}$ & $\mathrm{c}$ & 0.322789 & 0.332000 & 0.601389 \\
16 & $\mathrm{OH}$ & $\mathrm{c}$ & 0.990734 & 0.999945 & 0.261445 \\
17 & $\mathrm{Si}$ & $\mathrm{c}$ & 0.324068 & 0.666612 & 0.063936 \\
18 & $\mathrm{Si}$ & $\mathrm{c}$ & 0.657401 & 0.333278 & 0.063936 \\
19 & $\mathrm{O}$ & $\mathrm{s}$ & 0.324068 & 0.666611 & 0.270587 \\
20 & $\mathrm{O}$ & $\mathrm{s}$ & 0.657401 & 0.333279 & 0.270587 \\
21 & $\mathrm{O}$ & $\mathrm{s}$ & 0.490254 & 0.999946 & 0.000822 \\
22 & $\mathrm{O}$ & $\mathrm{s}$ & 0.990735 & 0.499465 & 0.000823 \\
23 & $\mathrm{O}$ & $\mathrm{s}$ & 0.491213 & 0.500424 & 0.000823 \\
24 & $\mathrm{OH}$ & $\mathrm{s}$ & 0.658484 & 0.999946 & 0.592063 \\
25 & $\mathrm{OH}$ & $\mathrm{s}$ & 0.990736 & 0.667694 & 0.592072 \\
26 & $\mathrm{OH}$ & $\mathrm{s}$ & 0.322984 & 0.332194 & 0.592072 \\
27 & $\mathrm{OH}$ & $\mathrm{s}$ & 0.990735 & 0.999945 & 0.268871 \\
\hline & & & & &
\end{tabular}

lution of the free energy as a function of the cell volume. The bulk modulus $\left(B_{0}\right)$ is a parameter describing the hardness of the material. By definition, it writes:

$B_{0}=V \frac{\partial^{2} F}{\partial V^{2}}$

The value of $B_{0}$ can be obtained by fitting $F$ as a function of the unit cell volume $V$ around the equilibrium volume $V_{0}$ by the Murnaghan equation of state (Murnaghan 1994; Pisani 1996) (see Fig. 2):

$$
\begin{gathered}
F=\frac{V_{0} B_{0}}{B_{0}^{\prime}}\left[\frac{1}{B_{0}^{\prime}-1}\left(\frac{V_{0}}{V}\right)^{B_{0}^{\prime}-1}+\left(\frac{V}{V_{0}}\right)\right]+C t e \\
\text { with } C t e=E_{0}-\frac{B_{0} V_{0}}{B_{0}^{\prime}-1}
\end{gathered}
$$

In the above equation, $F$ is the Helmoltz free energy, $B^{\prime}{ }_{0}$ is the derivative of $B_{0}$ with respect to pressure. The simulated bulk modulus of lizardite is $87 \mathrm{GPa}\left(B_{0}^{\prime}{ }_{0}\right.$ being equal to 3.3). When using the Voigt definition of the bulk modulus based on elastic constants (Gale and Rohl 2003):

$B_{0}^{\text {Voigt }}=\frac{1}{9}\left(C_{11}+C_{22}+C_{33}+2\left(C_{12}+C_{13}+C_{23}\right)\right)$

(where $C_{i j}$ are elastic constants given in Table 4), we found $B_{0}=82 \mathrm{GPa}$; a value that is indeed consistent with the one obtained with the Murnaghan equation of state. When using Reuss definition of $B_{0}$ from compliance matrix elements (Gale and Rohl 2003) given in
Table 5 , one obtains a lower bound value $B_{0}=40 \mathrm{GPa}$. Note that the compliance matrix is the inverse of the elastic constant matrix (Nye 1957):

$B_{0}^{\text {Reuss }}=\left(S_{11}+S_{22}+S_{33}+2\left(S_{12}+S_{13}+S_{23}\right)\right)^{-1}$

The Hill definition is the average of the two (Gale and Rohl 2003). It gives $B_{0}=61 \mathrm{GPa}$, a value that seems at first sight in good agreement with the experimental data of Tyburczy et al. (1991) for their low pressure phase of serpentine $B_{0}=63.5 \pm 3.5 \mathrm{GPa}$ obtained using shock wave experiments. The use of Hill route for bulk modulus determination may not be meaningful in the context of anisotropic layered materials such as lizardite. Note that the additional calculation for quartz with the same set of potential parameters for $\mathrm{O}$ and $\mathrm{Si}$ shows that Reuss and Voigt routes give very close results for the bulk modulus 47.2 and $46.8 \mathrm{GPa}$, respectively. Simulated (or experimental) values of lizardite bulk modulus are significantly larger than that of micas such as muscovite $\left(B_{0}=48 \mathrm{GPa}\right.$; Catti et al. 1994), phengite $\left(B_{0}=56 \mathrm{GPa} ; \quad\right.$ Pavese et al. 1999), Na-muscovite $\left(B_{0}=54 \mathrm{GPa}\right.$; Commodi and Zanazzi 1995), and phlogopite $\left(B_{0}=58 \mathrm{GPa}\right.$; Hazen and Finger 1978). Lizardite bulk modulus is lower than that of magnesium containing chlorite $\left(B_{0}=83 \mathrm{GPa}\right.$ for clinochlore, Welch and Crichton 2002). Clinochlore is known as being much stiffer along the $c$ direction compared to micas. Welch and Crichton (2002) proposed that the lower compressibility of clinochlore compared to micas arises 
Fig. 2 a Evolution of the Helmholtz free energy at room temperature $\mathrm{K}$ as a function of cell volume, when the structure is relaxed along directions $a$ and $c$ and isotropically $a c$, respectively. The inset displays the evolution of the Helmholtz free energy variations adjusted by the Murnaghan equation (see Eq. 13). b A zoom showing the evolution of the Helmholtz free energy as a function of cell volume, when the structure is relaxed along direction $c$

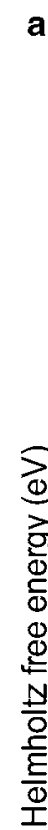
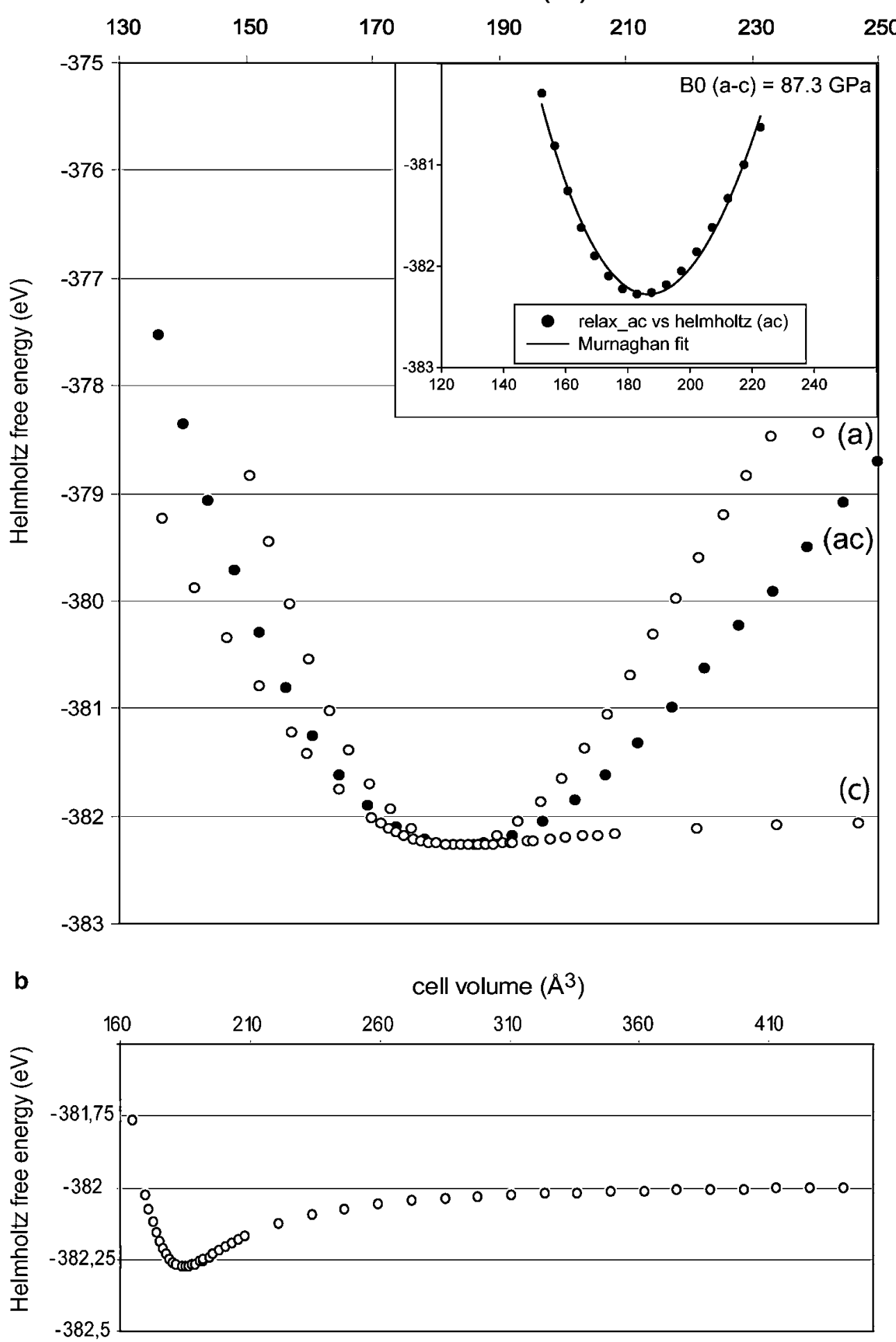

from the very different layer to layer bonding due to different occupancy of the interlayer space. Note that in chlorite, TOT silicate sheets have interlayer spaces occupied by a brucite-like octahedral sheet. The structure of both chlorites and serpentines is held by interlayer weak electrostatic interactions (often called hydrogen bonds, see below). As a consequence, similar elastic behavior of serpentines and chlorite may be expected. The anisotropy of the bulk modulus in lizardite structure can be understood looking at the shape energy wells when varying $a$ and $c$ unit cell parameters separately (see Fig. 2a, b). We obtained a value for $B_{0}$ of 144 and $29 \mathrm{GPa}$ in the $a$ and $c$ directions, respectively. As expected, lizardite is softer along the $c$ direction. 
Table 3 Comparison between the simulated and experimental structural properties of lizardite unit cell (at room temperature and 0 pressure)

\begin{tabular}{lcc}
\hline & Experimental structure $^{\mathrm{a}}$ & Modeled structure \\
\hline$a(\AA)$ & 5.338 & 5.434 \\
$c(\AA)$ & 7.257 & 7.153 \\
$V\left(\AA^{3}\right)$ & 179.1 & 184.0 \\
$\mathrm{Si}-\mathrm{O}_{\text {av }}(\AA)$ & 1.638 & 1.647 \\
$\mathrm{Mg}-\mathrm{O}_{\text {av }}(\AA)$ & 2.073 & 2.125 \\
$\mathrm{O}_{3}-\mathrm{H}_{3}(\AA)$ & & 0.891 \\
$\mathrm{O}_{4}-\mathrm{H}_{4}(\AA)$ & & 0.902 \\
\hline
\end{tabular}

${ }^{\mathrm{a}}$ From Mellini and Viti (1994)

Table 4 Calculated elastic constants (units $=\mathrm{GPa}$ )

\begin{tabular}{lcccccr}
\hline$i j$ indices 1 & 2 & 3 & 4 & \multicolumn{2}{l}{5} & \multicolumn{2}{l}{6} \\
\hline 1 & 229.08 & 89.044 & 13.558 & -0.0001 & 4.6025 & 0.0001 \\
2 & 89.044 & 229.08 & 13.557 & 0.0001 & -4.6016 & 0.0001 \\
3 & 13.558 & 13.557 & 45.838 & -0.0001 & 0.0015 & 0.0001 \\
4 & -0.0001 & 0.0001 & -0.0001 & 12.765 & -0.0001 & -4.4598 \\
5 & 4.6025 & -4.6016 & 0.0015 & -0.0001 & 12.774 & 0.0001 \\
6 & 0.0001 & 0.0001 & 0.0001 & -4.4598 & 0.0001 & 70.0166 \\
\hline
\end{tabular}

This was predicted by Mellini and Zanazzi (1989) who observed experimentally a higher decrease of lizardite $c$ parameter compared to $a$ as a function of pressure (see below).

Acoustic transverse wave velocity, $V_{\mathrm{t}}$, and longitudinal wave velocity, $V_{1}$, are key quantities in the interpretation of seismic data. They can be derived combining data for the bulk modulus but also that for the shear modulus $G_{0}$ and the material density $\rho\left(=2.5155 \mathrm{~g} / \mathrm{cm}^{3}\right)$.

$$
\begin{aligned}
G_{0}^{\mathrm{Voigt}}=\frac{1}{15}\left(C_{11}+C_{22}+C_{33}+3\left(C_{44}+C_{55}+C_{66}\right)\right. & \\
& \left.-C_{12}-C_{23}-C_{13}\right) \\
G_{0}^{\text {Reuss }}= & 15\left(4\left(S_{11}+S_{22}+S_{33}-S_{12}-S_{13}-S_{23}\right)\right. \\
& \left.+3\left(S_{44}+S_{55}+S_{66}\right)\right)^{-1}
\end{aligned}
$$

Then,

$V_{\mathrm{t}}=\sqrt{\frac{G_{0}}{\rho}}$

and
$V_{1}=\sqrt{\frac{4 G_{0}+3 B_{0}}{3 \rho}}$

Using $G_{0}$ and $B_{0}$ values, we found $V_{\mathrm{t}}=6.4 \pm 1.1 \mathrm{~km} / \mathrm{s}$ and $V_{1}=3.6 \pm 1.0 \mathrm{~km} / \mathrm{s}$ (note that error bars include data dispersion that arises from the use of Reuss or Voigt set of data). These data are to be compared with those reported in Christensen's (2004) paper that are around 6 and $2.5 \mathrm{~km} / \mathrm{s}$ for $V_{1}$ and $V_{\mathrm{t}}$, respectively. From transverse and longitudinal wave velocities, one can calculate the Poisson ratio using

$\sigma=\frac{V_{1}^{2}-2 V_{\mathrm{t}}^{2}}{2\left(V_{1}^{2}-V_{\mathrm{t}}^{2}\right)}$

We found $\sigma=0.265$, a value that compares favorably with that reported by Christensen (2004); additional calculations for quartz lead to a Poisson ratio of 0.15 markedly lower than that of lizardite as found in experiment (Christensen 2004).

We also calculated Young modulii, which describe the hardness of the material with respect to deformation. For each axis, this modulus is defined by the ratio of stress to strain:

$\gamma_{\alpha}=\frac{\sigma_{\alpha \alpha}}{\varepsilon_{\alpha \alpha}}$

Young modulii can be calculated from the elements of the compliance matrix (see Table 5). We found $\gamma_{x}=\gamma_{y}=S_{11}^{-1}=S_{22}^{-1}=188 \mathrm{GPa} \quad$ and $\gamma_{z}=S_{33}^{-1}=45 \mathrm{GPa}$. These results confirm that lizardite preferentially accommodates deformation along the $c$ direction.

Interaction energy between the layers

Another important result from this study is the possibility to assess the nature of the layer to layer interaction. Within the tetrahedral and the octahedral sheets, the structure is held by iono-covalent bonds. Recent atomistic modeling studies show that a good description of layer to layer interactions in phyllosilicates can be obtained using transferable potentials (Sainz-Diaz et al. 2001). In lizardite, the weak interactions which link the successive sheets in the $c$ direction are usually considered to be hydrogen bonding (Mellini 1982; Benco and Smrcok 1998). As already mentioned in the hydrogen

Table 5 Calculated compliance matrix (units $=\mathrm{GPa}^{-1}$ )

\begin{tabular}{llcclcl}
\hline$i j$ indices & 1 & 2 & 3 & 4 & 5 & 6 \\
\hline 1 & $5.267 \times 10^{-3}$ & $-2.045 \times 10^{-3}$ & $-9.540 \times 10^{-4}$ & 0.000000 & $-2.635 \times 10^{-3}$ \\
2 & $-2.045 \times 10^{-3}$ & $5.267 \times 10^{-3}$ & $-9.540 \times 10^{-4}$ & 0.000000 & 0.000000 \\
3 & $-9.540 \times 10^{-4}$ & $-9.540 \times 10^{-4}$ & $2.238 \times 10^{-2}$ & 0.000000 & $0.035 \times 10^{-3}$ & 0.000000 \\
4 & 0.000000 & 0.000000 & 0.000000 & $8.024 \times 10^{-2}$ & 0.00000 & 0.000000 \\
5 & $-2.635 \times 10^{-3}$ & $2.635 \times 10^{-3}$ & 0.000000 & 0.000000 & $8.018 \times 10^{-2}$ \\
6 & 0.000000 & 0.000000 & 0.000000 & $5.270 \times 10^{-3}$ & 0.000000000 \\
\hline
\end{tabular}


bonding process is not clearly defined in the general intermolecular theory; it results from a balance between electrostatic and short-range interactions. When gradually increasing the interlayer distance along the $c$ axis (and relaxing all atomic degrees of freedom at each stage), the Helmholtz free energy tends towards an asymptotic value that is twice the energy of one single isolated layer (see Fig. 2). The difference between the energy minimum and this value and this asymptotic value thus gives the layer to layer cohesive energy. According to our data, this cohesion energy between successive layers is $0.33 \mathrm{eV}(32 \mathrm{~kJ} / \mathrm{mol})$. Considering that in one lizardite cell, there are three outer hydroxyl groups, the average energy for one $\mathrm{O}-\mathrm{H}^{*} \mathrm{O}$ layer-tolayer bond is close to $11 \mathrm{~kJ} / \mathrm{mol}$, assuming the whole interlayer energy to originate from hydrogen bonds. This value is consistent with classical hydrogen bonding energies usually deduced from the (internal) energy of liquid water $(44 \mathrm{~kJ} / \mathrm{mol})$; each water molecule having between four and five neighbors. It is also fairly close to the $0 \mathrm{~K}$ value of $13 \mathrm{~kJ} / \mathrm{mol}$ obtained by Balan et al. (2002) who investigated lizardite structure with ab initio calculations. This result further confirms the reliability of the set of interatomic potentials used in this work in describing magnesium silica solids.

Simulation of lizardite as a function of pressure and temperature

We studied the effect of pressure and temperature on lizardite structure in the range of stability of serpentine minerals: external pressure from 0 to $10 \mathrm{GPa}$, and temperatures from 373 to $923 \mathrm{~K}$. The structure was relaxed with pressure steps between 0.1 and $0.5 \mathrm{GPa}$ and temperature steps of $50 \mathrm{~K}$. In Fig. 3, only data obtained at $373 \mathrm{~K}$ are reported; temperature having a very small effect on the structural behavior in agreement with the experimental data of Guggenheim and Zhan (1998). As expected, the $a$ and $c$ cell parameters decrease upon compression and pressure is mainly accommodated by the decrease of the $c$ parameter (see Fig. 3) in rather good agreement with the experiment. In particular, at $2 \mathrm{GPa}$, we find an axial compression ratio of 5:1 which is in agreement with the value given by Mellini and
Zanazzi (1989). The use of the third-order Birch-Murnaghan expression giving the unit cell volume variation as a function of the applied external pressure in hydrostatic conditions (Birch 1947) allows to obtain another estimation of the bulk modulus (and its derivative with respect to pressure):

$$
\begin{aligned}
P(V)= & \frac{3 B_{0}}{2}\left[\left(\frac{V_{0}}{V}\right)^{7 / 3}-\left(\frac{V_{0}}{V}\right)^{5 / 3}\right] \\
& \times\left\{1+\frac{3}{4}\left(B_{0}^{\prime}-4\right)\left[\left(\frac{V_{0}}{V}\right)^{2 / 3}-1\right]\right\}
\end{aligned}
$$

In Fig. 4, we show the fit of data at $373 \mathrm{~K}$ using the Birch-Murnaghan equation. The $B_{0}$ value is found at $52 \mathrm{GPa}$ in relatively good agreement with the Hill value (61 GPa) averaged from Reuss and Voigt values but significantly smaller than the value of $87 \mathrm{GPa}$ found using the Murnaghan equation (see Eq. 13). Following the Birch-Murnaghan route, $B_{0}{ }^{\prime}$ is found at 5.2.

While the evolution of the $a$ parameter is linear with pressure, a change of behavior for the $c$ parameter is evidenced around $2 \mathrm{GPa}$ (see Fig. 3). Above this pressure, the structure becomes less compressible. The linear and continuous evolution of the free energy (not shown) suggests that there is no first-phase transition of lizardite in this pressure-temperature domain. Considering that compression is mainly accommodated by reduction of the interlayer space, we investigated the behavior of the surface $\mathrm{O}-\mathrm{H}$ bonds lying in the interlayer void (Fig. 5). Up to $10 \mathrm{GPa}$, the $\mathrm{O}_{3}-\mathrm{H}_{3}$ bond length does not display any significant change, which is in agreement with experimental studies (Kleppe et al. 2003) in contrast to that expected for a typical hydrogen bond (Benco and Smrcok 1998). However, the angle that $\mathrm{O}_{3}-\mathrm{H}_{3}$ bond forms with respect to the normal direction to the layer significantly varies, showing a bending of the $\mathrm{OH}$ bonds upon pressure (see Fig. 4). From quasi-vertical up to $2 \mathrm{GPa}$, they start bending up reaching an angle shift of $9^{\circ}$ at $10 \mathrm{GPa}$. It appears that the structure can compress up to $2 \mathrm{GPa}$ by decreasing the interlayer space without affecting the $\mathrm{OH}$ group geometry. Above this pressure, $\mathrm{OH}$ groups are forced to bend, implying a hardening of the structure. This bending was evocated in a recent paper dealing with the effect of pressure on serpentine
Fig. 3 Evolution of the $a$ and $c$ parameters (normalized to the corresponding room temperature and 0 pressure cell parameter) as a function of the applied external pressure.

Hollow circles (triangles) simulated (experimental) data along the $a$ direction, black circles (triangles) simulated (experimental) data along the $c$ direction. Experimental data from Melini and Zanazzi (1989)

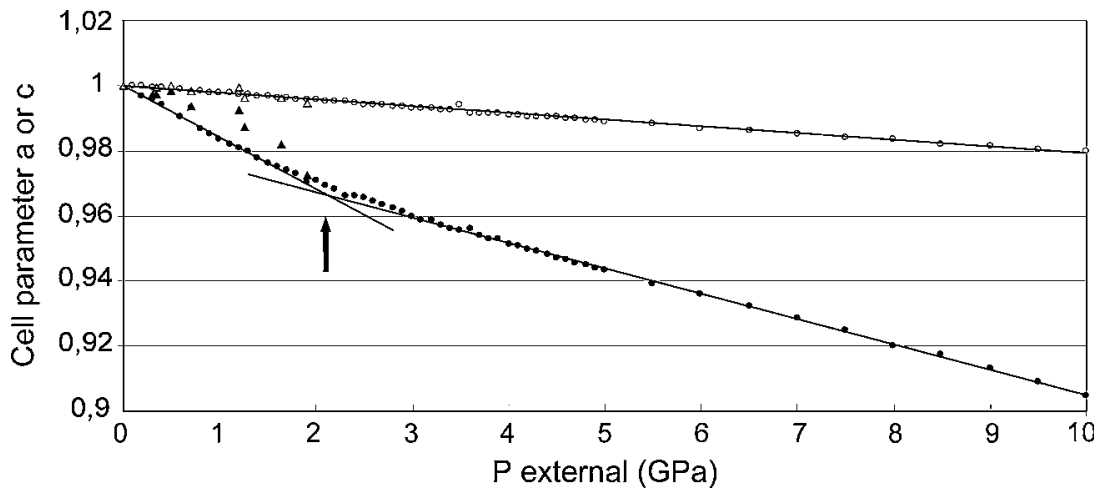




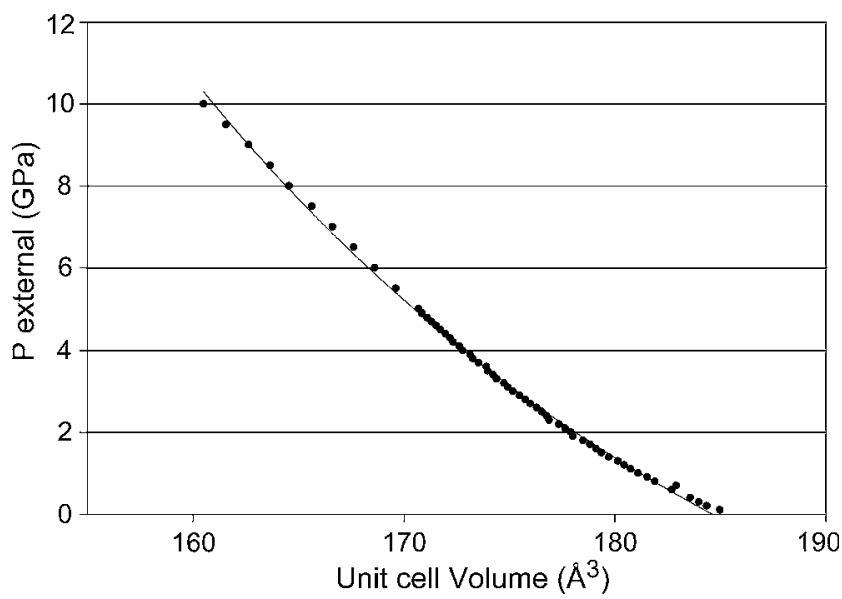

Fig. 4 Fit of PV data at $373 \mathrm{~K}$ using the Birch-Murnaghan equation (see Eq. 22)

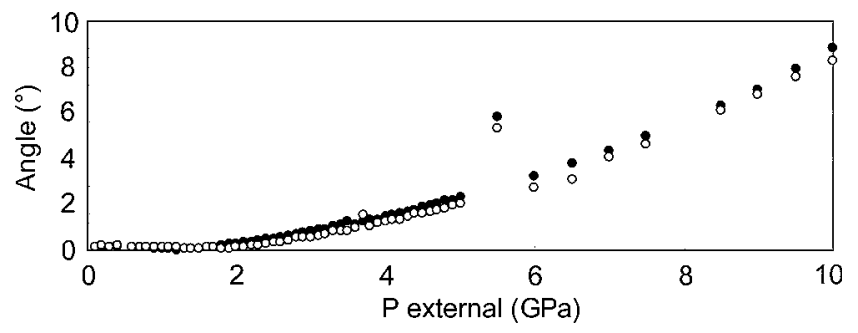

Fig. 5 Angle of interlayer $\mathrm{OH}$ bond with respect to the normal direction to the layers as a function of the applied external pressure. Hollow circles $373 \mathrm{~K}$, filled circles $823 \mathrm{~K}$

structures (Auzende et al. 2004) but the authors, on the basis of their data, rather explained Raman band shifts by a shortening of the $\mathrm{O}-\mathrm{H}$ bonds. These data could be reinterpreted at the light of the present study.

\section{Conclusion}

In this study, we checked the ability of ionic (shell-core) empirical potentials in describing the properties of serpentine minerals. Simulations of lizardite using the GULP code proved to yield reliable information. The simulated equilibrium structure of lizardite is in good agreement with the experimental one determined by Mellini and Viti (1994). The evolution of simulated lizardite under pressure and temperature is also in agreement with experiment. The calculated layer to layer interaction energy is compatible with $\mathrm{H}$ bonding and is in good agreement with ab initio calculations of Balan et al. (2002). In addition, our simulations correctly reproduced the anisotropy of compressibility of lizardite that is evidenced from high-pressure experiments. We thus conclude that serpentine minerals can be adequately modeled by ionic semi-empirical interatomic potentials. We also predict a bending of the interlayer $\mathrm{OH}$ groups for pressures larger than $2 \mathrm{GPa}$. This corresponds to a hardening of the structure at larger pressure. This study also allowed investigating lizardite elastic properties and calculating average bulk, shear, and Young modulii as well as acoustic wave longitudinal and transversal velocities; data that are needed in tectonophysics geophysical modeling of oceanic hydrated lithosphere.

Antigorite and chrysotile, the two other main serpentine varieties, display more complex unit cell and presently cannot be investigated by ab initio calculations. There is currently a lack of data for these minerals due to the complexity of their structures, the lack of suitable single crystals for $\mathrm{x}$-ray diffraction studies, and the high amount of structural defects encountered in their microstructures. Investigations of these phases by energy minimization calculation using the approach described in this work will provide insights on the relative stability of these serpentine varieties and their elastic properties.

Acknowledgments The authors thank the two anonymous reviewers whose constructive remarks permitted to improve the manuscript. We are also grateful to Dr. E. Balan (Institut de Minéralogie et de Physique des Milieux Condensés, Paris), and Pr. B. Grobety (Earth Sciences Department, University of Fribourg, Germany) for insightful discussions.

\section{References}

Archer TD, Birse SEA, Dove MT, Redfern SAT, Gale JD, Cygan RT (2003) An interatomic potential model for carbonates allowing for polarization effect. Phys Chem Miner 30:416-424

Auzende AL, Daniel I, Reynard B, Lemaire C, Guyot F (2004) High-pressure behaviour of serpentine minerals: a Raman spectroscopic study. Phys Chem Miner 31:269-277

Balan E, Saitta AM, Mauri F, Lemaire C, Guyot F (2002) First principles calculation of the infrared spectrum of lizardite. Am Mineral 87:1286-1290

Benco L, Smrcok L (1998) Hartree-Fock study of pressure-induced strengthening of hydrogen bonding in lizardite-1T. Eur $\mathrm{J}$ Mineral 10:483-490

Birch F (1947) Finite elastic strain of cubic crystals. Phys Rev 71:809-824

Capitani G, Mellini M (2004) The modulated crystal structure of the "odd", $m=17$, antigorite polysome. Am Mineral 89:147-158

Catti M, Ferraris G, Hull S, Pavese A (1994) Powder neutron diffraction study of $2 \mathrm{M} 1$ muscovite at room pressure and at 2 GPa. Eur J Mineral 6:171-178

Christensen N (2004) Serpentine, peridotites and seismology. Int Geol Rev 46:795-816

Collins DR, Catlow CRA (1992) Computer simulations of structure and cohesive properties of micas. Am Mineral 77:11721181

Commodi P, Zanazzi PF (1995) High pressure structural study of muscovite. Phys Chem Miner 22:170-177

De Leeuw NH, Watson JW, Parker SC (1995) Atomistic simulation of the effect of dissociative adsorption of water on the surface structure and stability of calcium and magnesium oxide. J Phys Chem 99:17219-17225

Dick BG, Overhauser AW (1958) Theory of the dielectric constants of alkali halide crystals. Phys Rev 112:90-103

Dove MT, Cool T, Palmer DC, Putnis A, Salje EKH, Winkler B (1993) On the role of $\mathrm{Al} / \mathrm{Si}$ ordering in the cubic-tetragonal phase transition in leucite. Am Mineral 78:486-492

Gale JD (1996) Empirical potential derivation for ionic materials. Phil Mag B 73:3-19 
Gale JD (1997) Gulp: a computer program for symmetry-adaopted simulation of solids. J Chem Soc Faraday Trans 93:629-637

Gale JD, Rohl AL (2003) The General Utility Lattice Program (GULP). Mol Simulat 29:291-341

Gregorkiewitz M, Lebech B, Mellini M, Viti C (1996) Hydrogen positions and thermal expansion in lizardite-1 $T$ from Elba: a low-temperature study using Rietveld refinement of neutron diffraction data. Am Mineral 81:1111-1116

Guggenheim S, Zhan W (1998) Effect of temperature on the structure of lizardite $1 \mathrm{~T}$ and 2H. Can Mineral 36:1587-1594

Hattori K, Guillot S (2003) Volcanic fronts as a consequence of serpentinite dehydration in the mantle wedge. Geology 31:525-528

Hazen M, Finger LW (1978) The crystal structures and compressibilities of layer minerals at high pressure; I, $\mathrm{SnS}<2$ ), berndtite. Am Mineral 63:289-292

Kleppe AK, Jephcoat AP, Welch MD (2003) The effect of pressure upon hydrogen bonding in chlorite: a Raman spectroscopic study of clinochlore to $26.5 \mathrm{GPa}$. Am Mineral 88:567-573

Mellini M (1982) The crystal structure of lizardite-1T: hydrogen bonds and polytypism. Am Mineral 67:587-598

Mellini M, Viti C (1994) Crystal structure of lizardite 1T. Am Mineral 72:1194-1198

Mellini M, Zanazzi PF (1989) Effects of pressure on the structure of lizardite 1T. Eur J Mineral 1:13-19

Murnaghan FD (1994) The compressibility of media under extreme pressures. Proc Natl Acad Sci 30:244-247

Nye JF (1957) Physical properties of crystals. Oxford University Press, Oxford

Palin EJ, Dove MT, Redfern SAT, Saint-Diaz CI, Lee WT (2003) Computational study of tetrahedral Al-Si and octahedral Al$\mathrm{Mg}$ ordering in phengite. Phys Chem Miner 30:293-304

Pavese A, Ferraris G, Pischedda V, Mezouar M (1999) Synchrotron powder diffraction study of phengite 3T from the DoraMaira massif: $P-V-T$ equation of state and petrological consequences. Phys Chem Miner 26:460-467
Pisani C (1996) Ab initio approaches to the quantum-mechanical treatment of periodic systems. Lect Notes Chem 67:47-75

Post JE, Burnham CW (1986) Modeling tunnel-cation displacements in hollandites using structure-energy calculations. Am Mineral 71:1178-1185

Press WH, Teukolsky SA, Vetterling WT, Flannery BP (1986) Numerical recipes in Fortran: the art of scientific computing. Cambridge University Press, Cambridge

Price GD, Parker SC (1988) Physical properties and thermodynamic behaviour of minerals. EKH Salie 225:613-638

Sainz-Diaz GI., Hernandez-Laguna A, Dove MT (2001) Modelling of dioctaedral 2:1 phyllosilicates by means of transferable empirical potentials. Phys Chem Miner 28:130-141

Sauer J, Ugliengo P, Garrone E, Saunders VR (1994) Theoretical study of van der Walls complexes at surface sites in comparison with the experiment. Chem Rev 94:2095-2160

Teppen BJ, Rasmussen K, Bersch PM, Miller DM, Schaffer L (1997) Molecular dynamics modelling of clay minerals. 1. Gibbsite, kaolinite, pyrophyllite, and beidellite. J Phys Chem B 101:1579-1587

Tyburczy JA, Duffy TS, Ahrens TJ, Lange MA (1991) Shock equation of state of serpentine to $150 \mathrm{GPa}$ : Implications for the occurrence of water in the Earth's lower mantle. J Geophys Res 96:18011-18027

Welch MD, Crichton WA (2002) Compressibility of clinochlore to $8 \mathrm{GPa}$ at $298 \mathrm{~K}$ and a comparison with micas. Eur J Mineral 14:561-565

Wicks FJ, O'Hanley D (1988) Serpentine minerals: structure and petrology. In: Bailey SW (ed) Reviews in mineralogy. Hydrous phyllosilicates, vol 19, pp 91-167

Winkler B, Dove MT, Leslie M (1991) Static lattice energy minimization and lattice dynamics calculations on aluminosilicate minerals. Am Mineral 76:313-331 